\title{
Discounted costs and obsolescence with the Joint replenishment problem
}

\author{
Ricardo Afonso $^{\text {a,b }}$, Pedro Godinho ${ }^{\mathrm{a}}$ and João Paulo Costa ${ }^{\mathrm{a}^{*}}$
}

${ }^{a}$ Univ Coimbra, CeBER, Faculty of Economics, Av Dias da Silva 165, 3004-512 Coimbra, Portugal

${ }^{b}$ Altice Labs, S.A., Rua Eng. Jose Ferreira Pinto Basto, 3810-106, Aveiro, Portugal

\section{H R O N I C L E}

Article history:

Received February 172021

Received in Revised Format

May 232021

Accepted July 282021

Available online

August, 42021

Keywords:

Inventory lot sizing

Joint replenishment problem

Obsolescence

\section{A B S T R A C T}

Real life inventory lot sizing problems are frequently challenged with the need to order different types of items within the same batch. The Joint Replenishment Problem (JRP) addresses this setting of coordinated ordering by minimizing the total cost, composed of ordering (or setup) costs and holding costs, while satisfying the demand. The complexity of this problem increases when some or all item types are prone to obsolescence. In fact, the items may experience an abrupt decline in demand because they are no longer needed, due to rapid advancements in technology, going out of fashion, or ceasing to be economically viable. This article proposes an extension of the Joint Replenishment Problem (JRP) where the items may suddenly become obsolete at some time in the future. The model assumes constant demand and the items' lifetimes follow independent negative exponential distributions. The optimization process considers the time value of money by using the expected discounted total cost as the minimization criterion. The proposed model was applied to some test cases, and sensitivity analyses were performed, in order to assess the impact of obsolescence on the ordering policy. The increase in the obsolescence risk, through the progressive increase of the obsolescence rates of the item types, determines smaller lot sizes on the ordering policy. The increase in the discount rate causes smaller quantities to be ordered as well.

\section{Introduction}

The Economic Order Quantity (EOQ) model is one the oldest models in the inventory lot sizing literature. Harris (1913) proposed the model under the assumption of constant demand, for a single item type, recognizing the need to balance ordering and holding costs. Another seminal single item contribution to this problem is due to Taft (1918, apud Holmbom \& Segerstedt, 2014) who extended the EOQ by incorporating a finite production rate, originating the well-known Economic Production Quantity (EPQ) model or Economic Production Lot (EPL) model. Since then, several new inventory lot sizing models have appeared in the literature, particularly those considering the inventory management of multiple item types, instead of just one, and also items prone to obsolescence.

The EOQ $\backslash E P Q$ based models are still widely accepted by many industrial sectors, especially due to their simplicity and effectiveness (Andriolo et al., 2014). However, these models have some restrictive assumptions as, for example, to consider a single type of item. Companies are frequently confronted with the need to order different items from a single supplier. Coordinated ordering may lead to a reduction on fixed costs, for example, by filling a truckload or by substantially lowering the setup costs if a group of products are manufactured together in a production line (Axsäter, 2015). In this setting, the Joint Replenishment Problem (JRP) encompasses the coordinated replenishment of different types of items in the same order (Khouja \& Goyal, 2008), generally provided by the same supplier, or with the determination of lot sizes and schedule of multiple types of items in single-facility production/inventory systems (Lee \& Yao, 2003).

* Corresponding author Tel.: +351239790586

E-mail: jpaulo@fe.uc.pt (J. P. Costa)

2022 Growing Science Ltd.

doi: $10.5267 /$ j.ijiec. 2021.8 .002 
The possibility of item obsolescence is also a relevant issue in several inventory lot sizing problems. As the point at which an item becomes obsolete cannot be predicted in advance, obsolescence is usually characterized by uncertainty in the product lifespan. This sort of items typically experiences an abrupt decline in demand because they are no longer needed, due to an advance in technology, going out of fashion, or ceasing to be economically viable. In other words, obsolescence typically occurs when an item has been superseded by a better version (Nahmias, 2011). As a consequence, obsolescence results in a partial or total loss of value of the inventory on hand (van Delft \& Vial, 1996). Thus, obsolescence is not alien to the literature of inventory lot sizing. However, despite the existence of literature addressing the obsolescence, most of it assumes a single item type approach, such as the studies of Masters (1991), Joglekar \& Lee (1993), Cobbaert \& Van Oudheusden (1996), Emsermann \& Simon (2007), Arcelus et al. (2006) and van Delft \& Vial (1996), among others.

Khouja \& Goyal (2008) point out that JRP models are vastly more applicable in real life situations than inventory models assuming a single item type and advocate more research on practical extensions of the JRP, namely to explore the JRP dealing with items subject to obsolescence. In a more recent JRP literature review, Bastos et al. (2017) also mention relevant research on extensions and practical applications of the JRP, but obsolescence is not referenced at all. To our best knowledge, models considering items prone to obsolescence under the JRP are still not available in the literature.

This article proposes an inventory lot sizing model involving multiple types of items subject to obsolescence under the framework of the Joint Replenishment Problem (JRP). The type of obsolescence to be addressed in the present article is the sudden obsolescence, which occurs when the product design or system specifications change and existing inventories (of items, products or their parts) are no longer needed (Sandborn, 2013).

The model developed in this study uses the single-item total obsolescence model proposed by van Delft \& Vial (1996) as a starting point and extends it to the case of multiple item types under the JRP. Both the van Delft \& Vial (1996) and the classic JRP models use assumptions of the EOQ: constant demand, no quantity discounts, no shortages allowed, linear holding cost, and instantaneous delivery. The lead time is assumed to be zero. Obsolescence time, or the lifetime of the item types, follows a negative exponential distribution. According to Masters (1991), "although many distributions are plausible, the (negative) exponential (distribution) is appropriate for sudden obsolescence phenomenon since it models a constant obsolescence rate", i.e. "the age of the item does not influence the probability of obsolescence during any subsequent interval", which means that the obsolescence process has no memory and a time-invariant lot size is optimal. As van Delft \& Vial (1996), we also incorporate the time value of money in the proposed model by considering the firm discount rate.

This article is structured as follows. Section 2 characterizes and presents the formulation of the classical JRP while section 3 discusses literature that addresses the characteristics of obsolescence and presents a literature review on inventory lot sizing where items are prone to obsolescence. In section 4, we first present the total obsolescence single-item model developed by van Delft \& Vial (1996). We include the holding cost per item and per unit of time, which was not originally considered by van Delft and Vial. Then, we develop an extended model to cope with multiple types of items in the context of the JRP. The model formulation for the general case involving item types is presented, and an iterative and recursive procedure for performing the calculations is also proposed. Some numerical examples and results are presented and discussed in section 5.

We can conclude that optimal order quantities of coordinated replenishments of multiple obsolescent item types are smaller than the quantities of items that are not subject to obsolescence. This is in line with the results obtained by van Delft \& Vial (1996) with the single-item model. As far as the savings of the coordinated ordering are concerned, they decrease when the obsolescence rates increase, and they become more prominent with higher major setup costs.

The number of cost components of the function to be optimized increases with the number of item types considered in the problem. This may lead to large sized problems. However, because the value of some cost components approaches zero as the number of item types increases, we may consider the possibility of simply removing these components from the calculations.

In section 6, some conclusions are summarized.

\section{Inventory lot sizing and the Joint Replenishment Problem}

The JRP considers the coordinated replenishments of different types of items in the same order (Khouja \& Goyal, 2008), and so it has potential applications whenever a family of items can be defined on the basis of a common supplier, common mode of transportation or common production facility (Webb et al., 1997). Typically, the objective is to minimize the total cost, composed of ordering (or setup) costs and holding costs, while satisfying the demand. The JRP can be interpreted as an extension of the classical EOQ, which considers the optimal trade-off between order costs and holding costs for a single type of items but extended into a more complex setting involving multiple item types. The increase in complexity turns the JRP into a NP-hard problem, meaning that it is unlikely that a polynomial time algorithm to solve the JRP exists (Cohen \& Yedidsion, 2015). Most of the assumptions of the classic JRP are similar to those of the classical EOQ, including constant demand, no quantity discounts, no shortages allowed, linear holding cost, and instantaneous delivery (i.e. replenishment rate is infinite). Without loss of generality, the lead time is equal to zero. However, the cost of placing an order to the supplier includes two components, one major ordering cost (or major setup cost) independent of the number of different items in the order, and several minor ordering costs (or minor setup costs) that depend on the number of different types of items included 
in the order (Khouja \& Goyal, 2008). The assumptions underlying the classical EOQ imply that it is optimal to place orders only when the inventory level hits zero (Berk \& Gurler, 2017). In the JRP context, it is reasonable to consider a (cyclic) policy which includes a base cycle $T$ and a set of integer multipliers $k_{i}$ such that the time interval between successive replenishments of item type $i$ is given by $T_{i}=k_{i} T$. This means that an item type $i$ where $k_{i}=1$ is included in each and every order. Two types of strategies can be generally applied to the JRP: a direct grouping strategy (DGS) and an indirect grouping strategy (IGS). According to the DGS, the different item types are separated into a predetermined number of sets and the item types within each set are jointly replenished. Under IGS, a replenishment is made at regular time intervals (every $T$ units of time) and each item type $i$ has a replenishment quantity sufficient to last for exactly an integer multiple $k_{i}$ of $T$ (Li et al., 2009).

\subsection{Notation and formulation of the classic JRP}

Consider the following notation:

$$
\begin{aligned}
& N \text { - number of item (or product) types; } \\
& i-1,2, \ldots, N \text { - the item (or product) type index; } \\
& C \text { - total ordering and holding costs per unit of time; } \\
& T \text { - time between successive replenishments (or base cycle) expressed in units of time; } \\
& A \text { - major ordering cost (major setup cost) associated with each replenishment; } \\
& a_{i} \text { - minor ordering cost incurred if item type } i \text { is ordered in a replenishment; } \\
& h_{i} \text { - unit holding cost for item type } i \text {, per unit of time; } \\
& D_{i} \text { - demand of item type } i \text { per unit of time; } \\
& T_{i} \text { - time interval between successive replenishments of item type } i ; \\
& Q_{i} \text { - order quantity of item type } i \text { (quantity of items of type } i \text { to order); } \\
& C_{O} \text { - ordering costs per unit of time; } \\
& C_{H} \text { - holding costs per unit of time. }
\end{aligned}
$$

Under IGS the cycle time of every item type is an integer multiple $k_{i}$ of the base cycle $T$ such that $T_{i}=k_{i} T$ and the corresponding order quantity is given by $Q_{i}=T_{i} D_{i}=T k_{i} D_{i}$.

Following Khouja \& Goyal (2008), the ordering costs per unit of time are

$$
C_{O}=\frac{A}{T}+\sum_{i=1}^{N} \frac{a_{i}}{k_{i} T}=\frac{A+\sum_{i=1}^{N} \frac{a_{i}}{k_{i}}}{T}
$$

and the holding costs per unit of time are

$$
C_{H}=\sum_{i=1}^{N} \frac{Q_{i} h_{i}}{2}=\frac{T}{2} \sum_{i=1}^{N} k_{i} D_{i} h_{i} .
$$

Thus, the total costs per unit of time are

$$
C=C_{O}+C_{H}
$$

We should be aware that the major setup cost still occurs in cycles with no replenishments (i.e. $k_{\mathrm{i}} \geq 2, \mathrm{i}=1,2, \ldots, \mathrm{N}$ ). This situation is dealt with by Dagpunar (1982) and Porras and Dekker (2008).

\section{Obsolescence in the Joint Replenishment Problem}

The classical EOQ and JRP models implicitly assume that the stocked items have infinite shelf lives. However, there are situations where the goods in stock may deteriorate (e.g. pharmaceuticals, alcohol) or become obsolete (e.g. fashion), thus being subject to losses of their value over time. 
According to Goyal \& Giri (2001) and Bakker et al. (2012) deterioration refers to the damage, spoilage, dryness, vaporization, etc. of the products. On the other side, items are subject to obsolescence if they lose their value over time because of rapid changes of technology or the introduction of a new product by a competitor, or because they go out of fashion (Goyal \& Giri, 2001; Khanlarzade et al., 2014). In the context of obsolescence, Pahl and Vo $\beta$ (2014) consider the products whose functionality does not degrade, but where demand deteriorates over time as customers' perceived utility decreases. Sandborn (2013) and Bartels et al. (2012) consider two types of obsolescence: procurement obsolescence or Diminishing Manufacturing Sources and Material Shortages obsolescence - DMSMS and sudden obsolescence of inventory obsolescence. The DMSMS is concerned with the unavailability of technologies or parts (where "part" refers to the lowest management-manufacturing, sparing, repairing level for the system) that are necessary to manufacture or sustain a system. The type of obsolescence to be addressed in the present article is the sudden obsolescence, which occurs when the product design or system specifications change and, as a result, existing inventories (of products or their parts) are no longer needed. In other words, sudden obsolescence may occur because the functions served by an item render it useless, because it is replaced by a substitute one performing similar or identical functions, or the like (Arcelus et al., 2002). Obsolescence typically occurs when an item has been superseded by a better version (Nahmias, 2011). As a consequence, obsolescence results in a partial or total loss of value of the inventory on hand (van Delft \& Vial, 1996). At the point in time when obsolescence occurs, it is typically assumed that the demand drops suddenly to zero. This discontinuity in demand distinguishes deterioration from obsolescence. Sudden obsolescence occurs when the utility loss is relatively abrupt and demand collapses overnight. The point at which an item becomes obsolete cannot be predicted in advance. Hence, obsolescence is typically characterized by uncertainty in the lifetime of a product (Nahmias, 2011).

Song \& Zipkin (1996) investigate the impact of obsolescence on the inventory policy and conclude that significant savings can be obtained by incorporating the risk of obsolescence in inventory decisions. The traditional approach to deal with obsolescence of items in inventory lot sizing problems is to add a risk component to the holding cost. Then, the optimal time between successive replenishments is derived using the EOQ model (Joglekar \& Lee, 1993). Cobbaert \& Van Oudheusden (1996) discuss obsolescence in an EOQ setting where different situations of obsolescence risk are studied under scenarios where shortage is allowed and under scenarios in which it is not allowed. Because the nature of the obsolescence cost is different from the holding costs, i.e. it is incurred only once and for all while the holding costs are incurred over a specified time interval (Oguji, 2018), van Delft \& Vial (1996) consider an expected discounted cost criterion to avoid using an ad hoc obsolescence cost.

In the study presented by Dohi \& Osaki (1995), the lifetime distribution of an item follows a generic and arbitrary probability distribution. Without discarding other plausible distributions, Masters (1991) argues that the negative exponential probability distribution is appropriate for modelling the lifetime of an item subject to sudden obsolescence. Considering the 'memoryless' property of the negative exponential distribution, this means that the age of the item does not influence the probability of obsolescence during the subsequent intervals. Masters (1991) and Joglekar \& Lee (1993) use the average cost criterion to determine the optimal solution for their sudden obsolescence models, but Joglekar \& Lee (1993) present an exact solution using a probabilistic analysis based on whether obsolescence occurs during or after an inventory cycle. Van Delft \& Vial (1996) perform a probabilistic analysis assuming a negative exponential distribution for the item lifetime.

The aforementioned studies on obsolescence consider constant demand, but other types of demand also exist. Menipaz (1986) considers that demand for successive units of the item occurs in accordance with a Poisson process. In Arcelus et al. (2002, 2006) the demand depends on the retailer's sale price. Under the assumption of stochastic-demand, Emsermann \& Simon (2007) consider the situation of determining the optimal ordering and reordering policy for an inventory in which the entire stock will simultaneously become obsolete at some (typically random) future time defined by a cumulative distribution function. Pierskalla (1969) presents a model under a finite and discrete-time planning horizon situation where it may be possible to state a probability mass function which gives the probability of an item to become obsolete in each and every period. In the view of the author, this function corresponds to a random variable which defines the horizon time.

Because high technology industries face decreasing component unit costs, Khouja et al. (2006) develop models for products which experience continuous unit cost decrease and are also subject to sudden obsolescence. Minimization of costs is not the only objective we can find in the literature, but profit maximization can also be found, as discussed in Arcelus et al. (2002, 2006). In another perspective, Dohi \& Osaki (1995) present a model where the objective is the maximization of the stationary availability, which is the probability that the stock is not depleted in the steady-state.

Thompson (1975) and van Delft \& Vial (1996) develop models with items subject to obsolescence by considering the time value of money where methods of capital budgeting are applied to the determination of optimal inventory levels.

In a literature review of the JRP, Khouja \& Goyal (2008) advocate the development of research on extensions and practical applications of the problem, which should continue receiving attention. Bastos et al. (2017) verified a trend in this direction. One of the extensions explicitly mentioned by Khouja \& Goyal (2008) has to do with including products facing obsolescence or deterioration in the JRP, but from Bastos et al. (2017) we can conclude that there continues to exist a lack of research in this field. In this line, Kouki et al. ( 2016) state that despite the JRP having been broadly studied in the literature, the coordinating replenishment of multiple deteriorating items is still unexplored. The challenge stemming from the limited lifetime of this type of item is pointed out as a possible cause. Nevertheless, some models considering the JRP together with items subject to deterioration (not obsolescence) exist in the literature, particularly for the case where the rate of deterioration 
is constant (Taleizadeh et al., 2013). Ai, Zhang, \& Wang (2017) approach the JRP by considering multiple non-instantaneous deteriorating item types. Kouki et al. (2016) consider in their JRP model a constant deterioration rate with stochastic demand. The authors suggest that a random lifetime following a negative exponential distribution is suitable for real situations where item lifetimes are typically small but occasionally long. Considering deterioration as a decaying process, exponential decay can be derived by assuming that a fixed fraction of stock on hand, given by the constant deterioration rate, is lost each period regardless of the age distribution of inventory (Nahmias, 1982). Li et al. (2009) present a JRP model where deterioration is interpreted in this context.

Now that we have reached this point, we find that some relevant models do exist in the literature where items or products are subject to obsolescence in inventory lot sizing decision problems, particularly models considering a single item type. However, when searching for JRP extensions considering multiple item types, we realize a lack of research in this field. In this way, the next section proposes a model that combines the JRP and items prone to obsolescence.

\section{The proposed model}

As explained before, the literature focusing on inventory lot sizing problems where items are subject to obsolescence typically only considers a single item type. Van Delft \& Vial (1996) models consider the impact of obsolescence for a single item type under the discounted cost criterion. According to the authors the use of this criterion is suitable to deal with the specific nature of the obsolescence cost, avoiding the use of an ad hoc obsolescence cost. While the obsolescence cost is incurred once and for all, the other costs, such as ordering and holding costs, occur during every cycle (Oguji, 2018). We take the van Delft and Vial (1996) single item type model as a starting point to develop a JRP based model where multiple item types are prone to obsolescence.

\subsection{The single-item type obsolescence model}

\section{Assumptions and notation}

Van Delft and Vial (1996) present a chain of single-item type obsolescence models where assumptions change from model to model. The first model proposed by van Delft and Vial (1996) considers total loss at obsolescence, which means that obsolescence is sudden and all items on hand lose their value at once, and also uses some of the assumptions of the classical EOQ model: instantaneous delivery, shortages are not allowed, constant demand rate, constant unit cost, linear holding cost and fixed ordering cost. Van Delft and Vial (1996) also assume the lead time to be zero. For convenience, we represent the ordering cost by the sum of two components: a major and a minor ordering cost. Additionally, the model considers an appropriate internal discount rate of the firm. The item lifetime is random and follows a negative exponential distribution. Thus, the time at which obsolescence occurs is unknown.

Let us consider the following notation:

$$
\begin{aligned}
& T \text { - time between successive replenishments (or base cycle) expressed in units of time; } \\
& Q \text { - order lot size (quantity of items to order); } \\
& A \text { - major ordering cost (major setup cost) associated with each replenishment; } \\
& a \text {-minor ordering cost (minor setup cost) associated with including the item in the order; } \\
& c \text { - unit cost of item (\$/unit); } \\
& h-\text { unit holding cost per unit time (\$/unit/time); } \\
& V(T) \text { - total discounted cost; } \\
& E(V(T)) \text { - total expected discounted infinite horizon cost; } \\
& D-\text { demand of the item per unit of time; } \\
& \delta \text { - discount rate; } \\
& \theta-\text { rate of obsolescence of the item. }
\end{aligned}
$$

The total discounted cost for an infinite time horizon, without considering obsolescence or holding costs, is expressed by (van Delft \& Vial, 1996):

$$
V(T)=\frac{A+a+c D T}{1-e^{-\delta T}}
$$

Total loss at obsolescence model with holding costs

Van Delft and Vial (1996) state that the structure of the optimal policy of the single-item type obsolescence model can be specified a priori. In fact, one must note that under instantaneous delivery, an order is placed only when the inventory level is zero. Thus, the ordering policy can be outlined by a sequence of ordering moments. Indeed, assuming that the inventory level is zero at some time $\mathrm{t}$ and that the corresponding optimal lot size is $Q$ such that $T=\frac{Q}{D}$, if the obsolescence does not occur 
during the period $\left[t, t+\frac{Q}{D}\right]$, the inventory will be inevitably zero at moment $t+\frac{Q}{D}$. As the obsolescence lifetime follows a memoryless distribution, the inventory system is in the same status at time $t+\frac{Q}{D}$ as it was at time $t$, maintaining $Q$ as the optimal lot size. To compute the total expected discounted cost, van Delft \& Vial (1996), in a similar way as Joglekar \& Lee (1993), separately analyze the cases where $t \leq T$ and $t>T$. If obsolescence occurs at time $t \leq T$, the incurred cost is the initial one, which is the sum of the ordering and items' acquisition costs, plus the expected discounted holding costs as determined at the beginning of the cycle. The ordering costs are $(A+a)$ and the items' acquisition costs are $(c D T)$. To compute the holding costs, we first realize that the inventory level at the beginning of the cycle is $Q$ units, and the inventory level at moment $t$ is $(Q-D t)=(D T-D t)$, as $Q=D T$. Then, the total holding costs are given by:

$$
h \int_{0}^{T}(D T-d t) d t=h\left(D T^{2}-\frac{1}{2} D T^{2}\right)=\frac{1}{2} h D T^{2}
$$

The corresponding discounted costs are given by:

$$
h \int_{0}^{T} e^{-\delta t}(D T-d t) d t
$$

Taking the expectation of (6) based on the negative exponential distribution of the item lifetime, the holding costs, as expected at the beginning of the cycle, are finally given by (see demonstration in Appendix A):

$$
h \int_{0}^{T} e^{-\delta t}(D T-D t) \theta e^{-\theta t} d t=h \theta\left(\frac{D T}{\delta+\theta}+\frac{D\left(e^{-(\delta+\theta) T}-1\right)}{(\delta+\theta)^{2}}\right)
$$

In a nutshell, when the obsolescence occurs at time $t \leq T$, the costs incurred at the beginning of the cycle are given by $A+$ $a+c D T+h \theta\left(\frac{D T}{\delta+\theta}+\frac{D\left(e^{-(\delta+\theta) T}-1\right)}{(\delta+\theta)^{2}}\right)$.

For the case where obsolescence occurs at a time $t>T$, we need to add the sum of the expected discounted costs, $E[V(T)]$, to be incurred after time $T$ (van Delft \& Vial, 1996). This sum must be discounted by the factor $e^{-\delta T}$. Hence, taking the expected value of all components, the total expected discounted costs are given by:

$$
E[V(T)]=A+a+c D T+h \theta\left(\frac{D T}{\delta+\theta}+\frac{D\left(e^{-(\delta+\theta) T}-1\right)}{(\delta+\theta)^{2}}\right)+e^{-(\theta+\delta) T} E[V(T)]
$$

Note that the term $e^{-\theta T}$ applied in the last component, $e^{-\delta T} E[V(T)]$, is the probability for the item to survive during the cycle $T$. In fact, it can be interpreted as a 'weight' $(<1)$ associated to a component which may not occur. No probability smaller than one is applied to the first component because the corresponding expected costs are assumed to be incurred with certainty as of the beginning of the cycle. The expression of $E[V(T)]$ can be rewritten as (see demonstration in Appendix B):

$$
E[V(T)]=\frac{A+a+c D T+h \theta\left(\frac{D T}{\delta+\theta}+\frac{D\left(e^{-(\delta+\theta) T}-1\right)}{(\delta+\theta)^{2}}\right)}{\left(1-e^{-(\delta+\theta) T}\right)}
$$

Huang (2004) proves in his study that, if we ignore the holding costs term, $E[V(T)]$ is convex. However, convexity can also be proved to the cost function expressed in (9). The demonstration is provided in Appendix C.

\subsection{Extension to JRP total loss obsolescence model}

The total loss obsolescence model proposed by van Delft \& Vial (1996) for a single item type is extended in this section in the light of the JRP context in order to coordinate the replenishment of multiple item types.

\section{Assumptions and notation}

The assumptions considered for the single-item model are kept for our total loss obsolescence JRP extended model, which coordinates the ordering of multiple item types. The lifetimes of the multiple item types are assumed to be independent and identically distributed and to follow a negative exponential distribution.

As discussed for the single-item obsolescence model, it makes sense to include an item type in a replenishment only when its inventory level is zero. Consequently, as argued for the classic JRP, it is reasonable to consider a replenishment policy which consists of a common base cycle and a set of integer multipliers, one multiplier for each item type, which corresponds to the number of base cycles between replenishments of the corresponding item type (Khouja \& Goyal, 2008; Silver et al., 2017).

The notation of the proposed model is:

$N$ - number of item (or product) types;

$i-1,2, \ldots, N-$ the item (or product) type index;

$S$ - the ordered set of $N$ item types; 
$T$ - time between successive replenishments (or base cycle) expressed in units of time involving all the $N$ item types;

$A$ - major ordering cost (major setup cost) associated with each replenishment;

$a_{i}$ - minor ordering cost incurred if item type $i$ is ordered in a replenishment;

$h_{i}$ - unit holding cost for item type $i$, per unit of time;

$D_{i}$ - demand of item type $i$ per unit of time;

$T_{i}$ - time interval between successive replenishments of item type $i$;

$k_{i}$ - integer multiplier such that $T_{i}=k_{i} T, i=1, \ldots, N$;

$B$ - a nonempty ordered subset of $S$ (i.e. $B \subseteq S$ and $B \neq \emptyset$ );

$\Gamma(B)$ - time between successive replenishments (or base cycle) expressed in units of time, but involving only the item types that do belong to $B$;

$k(B)$ - set of integer multipliers such that if $B=\left(i_{1}, i_{2}, \ldots, i_{m}\right)$ then $k(B)=\left(k_{i_{1}}, k_{i_{2}}, \ldots, k_{i_{m}}\right)$ where the multiplier $T_{i_{r}}=$ $k_{i_{r}} T, r=1, \ldots, m$

$Q_{i}$ - order quantity of item type $i$ (quantity of items of type $i$ to order);

$\delta$ - discount rate;

$\theta_{i}$ - rate of obsolescence of item type $i$;

$C_{o}$ - setup costs incurred during base cycle;

$C_{a}$ - acquisition costs incurred during base cycle;

$C_{h}$ - holding costs incurred during base cycle;

$\Psi_{0}$ - expected cost incurred during the first base cycle;

$\Psi_{n}$ - expected costs where $n$ item types do not become obsolete during the base cycle $T$ or, in other words, where $n$ of $N$ the item types survive the base cycle $T$, such that $(1 \leq n \leq N)$;

$V(B, \Gamma(B), k(B))$ - total discounted infinite horizon cost associated to item types $i$ that do belong to $B$;

$E[V(B, \Gamma(B), k(B))]$ - total expected discounted infinite horizon cost associated to item types $i$ that do belong to $B$.

When we refer to the optimal cost value of $E[V(B, \Gamma(B), k(B))]$, the notation appears with the symbol "*" at the top-right of $E$, i.e. $E^{*}[V(B, \Gamma(B), k(B))]$. The objective of the proposed model is to minimize $E[V(S, \Gamma(S), k(S))]=$ $E\left[V\left(S, T,\left(k_{1}, \ldots, k_{N}\right)\right)\right]$, i.e. find $E^{*}\left[V\left(S, T,\left(k_{1}, \ldots, k_{N}\right)\right)\right]$ where all the $N$ item types are involved. In a similar way as showed for the single-item case in (7), the holding costs of item type $i$ are given by:

$$
h_{i} \int_{0}^{T_{i}} e^{-\delta t}\left(D_{i} T_{i}-D_{i} t\right) \theta e^{-\theta_{i} t} d t=h_{i} \theta_{i}\left(\frac{D_{i} k_{i} T}{\delta+\theta_{i}}+\frac{D_{i}\left(e^{-\left(\delta+\theta_{i}\right) k_{i} T}-1\right)}{\left(\delta+\theta_{i}\right)^{2}}\right)
$$

For the derivation of the single-item expected discounted cost function, van Delft \& Vial (1996) analyze the cases where obsolescence occurs at time $t \leq T$ and $t>T$, i.e. during and after the base cycle $T$. In our proposed model, we consider an analogous approach where the obsolescence time occurs during or after the base cycle $T$.

\subsection{Costs incurred during the base cycle T}

Accordingly, the fixed order or setup costs and the costs of acquisition of the several items incurred at the beginning of the base cycle $T$ are given by $C_{o}=A+\sum_{i=1}^{N} a_{i}$ and $C_{a}=\sum_{i=1}^{N} C_{i} D_{i} k_{i} T$, respectively. The expected discounted holding costs incurred during the base cycle $T$ are given by $C_{h}=\sum_{i=1}^{N} h_{i} \theta_{i}\left(\frac{D_{i} k_{i} T}{\delta+\theta_{i}}+\frac{D_{i}\left(e^{-\left(\delta+\theta_{i}\right) k_{i} T}-1\right)}{\left(\delta+\theta_{i}\right)^{2}}\right)$. Then, the costs incurred during the base cycle are $\Psi_{0}=C_{o}+C_{a}+C_{h}$.

\subsection{Costs incurred after the base cycle $T$}

Considering now the case of costs incurred after the first base cycle, i.e. after moment $t=T$, we have to include the cost components with respect to all possible combinations where at least one item type survive the first base cycle $T$. For example, the cases where just one of the item types survives and the other $N-1$ do not, the cases where just two any item types survive and the other $N-2$ do not, and so on, until the last case where all the item types do survive the cycle $T$. Each of the cases is multiplied by the corresponding probabilities of survival and not survival of the individual item types. Moreover, each case is multiplied by a factor corresponding to the optimal expected discounted costs of the item types that survived. 
The total expected discounted costs must take into account the following terms:

- $\quad$ one term with the costs incurred during the first base cycle $T$ :

$\Psi_{0}=C_{o}+C_{a}+C_{h}$

- $\quad N$ terms with respect to the combinations where one item type survives the first base cycle $T$ :

$$
\Psi_{1}=\sum_{(i) \in S} E^{*}\left[V\left((i), \Gamma((i)),\left(k_{i}\right)\right)\right] e^{-\theta_{i} k_{i} T} \prod_{\substack{j \in S \\ j \neq i}}\left(1-e^{-\theta_{j} k_{j} T}\right)
$$

One must note that when optimizing $E\left[V\left((i), \Gamma((i)),\left(k_{i}\right)\right)\right]$, where there is only the product $i$ to consider, we logically set multiplier $k_{i}$ to one as we are not in a situation of coordinating the replenishments of different item types, but just one item type. Thus, optimizing the expected discounted costs with a single item type implies that we just have to use the van Delft \& Vial (1996) obsolescence model described in section 4.1.

- $\quad 2^{N}-N-2$ terms where $m(2 \leq m \leq N-1)$ types of items survive the first base cycle $T$ :

$$
\Psi_{m}=\sum_{\substack{B=\left(i_{1}, \ldots, i_{m}\right) \subset S \\ \operatorname{card}(B)=m}}\left(E^{*}[V(B, \Gamma(B), k(B))] e^{-\left(\theta_{i_{1}} k_{i_{1}}+\cdots+\theta_{i_{m}} k_{i_{m}}\right) T} \prod_{\substack{j \in S \\ j \notin B}}\left(1-e^{-\theta_{j} k_{j} T}\right)\right)
$$

This means that, for each $m, \Psi_{m}$ is the to the sum of $\left(\begin{array}{c}N \\ m\end{array}\right)=\frac{N !}{m !(N-m) !}$ components.

- $\quad$ one term where all the $N$ item types survive the first base cycle $T$ :

$$
\Psi_{N}=E[S, \Gamma(S), k(S)]\left(e^{-\left(\theta_{1} k_{1}+\ldots+\theta_{N} k_{N}\right) T}\right)=E\left[V\left(S, T,\left(k_{1}, \ldots, k_{N}\right)\right)\right]\left(e^{-\left(\theta_{1} k_{1}+\ldots+\theta_{N} k_{N}\right) T}\right)
$$

The number of terms in the expression of the general case is $2^{N}$ which is equal to one term with respect to the costs incurred during the first base cycle plus the number of terms with respect with the nonempty subsets of $S$ (i.e. the number of elements of $\wp(S)$ - the power set of $S$, minus the empty set). In other words, it is the term of costs incurred during the first base cycle plus the number of all the $n$-combinations of the $N$ item types, $n=1,2, \ldots, N$. Hence, the general expression of the total expected discounted cost is given by:

$$
\begin{gathered}
E\left[V\left(S, T,\left(k_{1}, \ldots, k_{N}\right)\right)\right]=\Psi_{0}+e^{-\delta T} E\left(\sum_{i=1}^{N} \Psi_{i}\right) \Leftrightarrow E\left[V\left(S, T,\left(k_{1}, \ldots, k_{N}\right)\right)\right]=\Psi_{0}+e^{-\delta T} E\left(\sum_{i=1}^{N-1} \Psi_{i}\right)+e^{-\delta T} \Psi_{N} \Leftrightarrow \\
E\left[V\left(S, T,\left(k_{1}, \ldots, k_{N}\right)\right)\right]-e^{-\delta T} \Psi_{N}=\Psi_{0}+e^{-\delta T} E\left(\sum_{i=1}^{N-1} \Psi_{i}\right) \\
\Leftrightarrow E\left[V\left(S, T,\left(k_{1}, \ldots, k_{N}\right)\right)\right]-e^{-\delta T}\left(e^{-\left(\theta_{1} k_{1}+\ldots+\theta_{N} k_{N}\right) T} E\left[V\left(S, T,\left(k_{1}, \ldots, k_{N}\right)\right)\right]\right)=\Psi_{0}+e^{-\delta T} E\left(\sum_{i=1}^{N-1} \Psi_{i}\right) \\
\Leftrightarrow E\left[V\left(S, T,\left(k_{1}, \ldots, k_{N}\right)\right)\right]=\frac{\Psi_{0}+e^{-\delta T} E\left(\sum_{i=1}^{N-1} \Psi_{i}\right)}{\left(1-e^{-\left(\delta+\theta_{1} k_{1}+\ldots+\theta_{N} k_{N}\right) T}\right)}
\end{gathered}
$$

\subsection{Simplification process for the expected total cost calculation}

As previously explained, the number of (all-positive) cost components in the $N$-item types total expected discounted infinite horizon cost expression is $2^{N}$. This may have a big impact in the number of calculations when facing a situation with a large number of item types. However, a more careful analysis can lead us to the simplification on the calculation of the total expected cost, especially by ignoring the cost terms which might be quite close to zero. In fact, the cost terms related to item types surviving the base cycle $T$ (i.e. $e^{-\delta T} E\left(\sum_{m=1}^{N-1} \Psi_{m}\right)$, when obsolescence occurs after the base cycle), decrease and approaches zero as the number of item types increase.

To compute the minimum of Eq. (15) it is necessary to consider an iterative and recursive procedure to determine the several $\Psi_{m}(m=1,2, \ldots, N-1)$, presented in Eq. (12) and Eq. (13). During the first iteration, we have to optimize each of the cases where just one of the items survive the first base cycle $T$ by applying the single-item model from van Delft \& Vial (1996). These values are needed to compute $\Psi_{1}$. In the second iteration, we have to optimize each of the cases where two of the item types survive the first base cycle $T$. The current iteration uses the optimal single-item total expected discounted costs determined in the previous one as input. That is to say, the values of $E^{*}\left[V\left(\left(i_{1}\right), \Gamma\left(\left(i_{1}\right)\right),\left(k_{i_{1}}\right)\right)\right]$ and 
$E^{*}\left[V\left(\left(i_{2}\right), \Gamma\left(\left(i_{2}\right)\right),\left(k_{i_{2}}\right)\right)\right]$ calculated in the first iteration are used as constants to compute $E^{*}\left[V\left(\left(i_{1}, i_{2}\right), \Gamma\left(\left(i_{1}, i_{2}\right)\right),\left(k_{i_{1}}, k_{i_{2}}\right)\right)\right]$ in the second iteration. All the expected costs involving any two item types are then used to compute $\Psi_{2}$. The procedure continues in this way until the last iteration in which $E^{*}\left[V\left(S, T,\left(k_{1}, \ldots, k_{N}\right)\right)\right]$ is finally computed. For example, let us consider the simplest case where $N=2$. In addition to the case in which the two items do become obsolete during the first base cycle, there are three more cases to take into account: only item type 1 survives, only item type 2 survives, and both item types survive. In this way, the expression of the expected discounted total cost where $S=$ $(1,2)$ is given by:

$$
\begin{aligned}
E\left[V\left(S, T,\left(k_{1}, k_{2}\right)\right)\right] & \\
= & A+a_{1}+a_{2}+c_{1} D_{1} T_{1}+c_{2} D_{2} T_{2}+h_{1} \theta_{1}\left(\frac{D_{1} T_{1}}{\delta+\theta_{1}}+\frac{D_{1}\left(e^{-\left(\delta+\theta_{1}\right) T_{1}}-1\right)}{\left(\delta+\theta_{1}\right)^{2}}\right) \\
+ & h_{2} \theta_{2}\left(\frac{D_{2} T_{2}}{\delta+\theta_{2}}+\frac{D_{2}\left(e^{-\left(\delta+\theta_{2}\right) T_{2}}-1\right)}{\left(\delta+\theta_{2}\right)^{2}}\right) \\
+ & e^{-\delta T}\left\{E^{*}\left[V\left((1), \Gamma((1)),\left(k_{1}\right)\right)\right]\left[e^{-\theta_{1} T_{1}}\left(1-e^{-\theta_{2} T_{2}}\right)\right]\right. \\
+ & \left.E^{*}\left[V\left((2), \Gamma((2)),\left(k_{2}\right)\right)\right]\left[e^{-\theta_{2} T_{2}}\left(1-e^{-\theta_{1} T_{1}}\right)\right]+\left[e^{-\left(\theta_{1} T_{1}+\theta_{2} T_{2}\right) T} E\left[V\left(S, T,\left(k_{1}, k_{2}\right)\right)\right]\right]\right\} \\
=A+a_{1}+a_{2}+ & c_{1} D_{1} k_{1} T+c_{2} D_{2} k_{2} T+h_{1} \theta_{1}\left(\frac{D_{1} k_{1} T}{\delta+\theta_{1}}+\frac{D_{1}\left(e^{-\left(\delta+\theta_{1}\right) k_{1} T}-1\right)}{\left(\delta+\theta_{1}\right)^{2}}\right) \\
& +h_{2} \theta_{2}\left(\frac{D_{2} k_{2} T}{\delta+\theta_{2}}+\frac{D_{2}\left(e^{-\left(\delta+\theta_{2}\right) k_{2} T}-1\right)}{\left(\delta+\theta_{2}\right)^{2}}\right) \\
& +e^{-\delta T}\left\{E^{*}\left[V\left((1), \Gamma((1)),\left(k_{1}\right)\right)\right]\left[e^{-\theta_{1} k_{1} T}\left(1-e^{-\theta_{2} k_{2} T}\right)\right]\right. \\
& \left.+E^{*}\left[V\left((2), \Gamma((2)),\left(k_{2}\right)\right)\right]\left[e^{-\theta_{2} k_{2} T}\left(1-e^{-\theta_{1} k_{1} T}\right)\right]+E\left[V\left(S, T,\left(k_{1}, k_{2}\right)\right)\right]\left[e^{-\left(\theta_{1} k_{1}+\theta_{2} k_{2}\right) T}\right]\right\} \Leftrightarrow
\end{aligned}
$$

$E\left[V\left(S, T,\left(k_{1}, k_{2}\right)\right)\right]$

$$
\begin{aligned}
& =\frac{A+\sum_{i=1}^{2}\left(a_{i}+c_{i} d_{i} k_{i} T+h_{i} \theta_{i}\left(\frac{D_{i} k_{i} T}{\delta+\theta_{i}}+\frac{D_{i}\left(e^{-\left(\delta+\theta_{i}\right) k_{i} T}-1\right)}{\left(\delta+\theta_{i}\right)^{2}}\right)\right)}{\left(1-e^{-\left(\delta+\theta_{1} k_{1}+\theta_{2} k_{2}\right) T}\right)} \\
& +\frac{\sum_{\substack { i=1 \\
\begin{subarray}{c}{j=1 \\
j \neq i{ i = 1 \\
\begin{subarray} { c } { j = 1 \\
j \neq i } }\end{subarray}}^{2} E^{*}\left[V\left((i), \Gamma((i)),\left(k_{i}\right)\right)\right]\left[e^{-\left(\delta+\theta_{i} k_{i}\right) T}\left(1-e^{-\theta_{j} k_{j} T}\right)\right]}{\left(1-e^{-\left(\delta+\theta_{1} k_{1}+\theta_{2} k_{2}\right) T}\right)}
\end{aligned}
$$

In this case, the procedure considers one iteration where $E^{*}\left[V\left((1), \Gamma((1)),\left(k_{1}\right)\right)\right]$ and $E^{*}\left[V\left((2), \Gamma((2)),\left(k_{2}\right)\right)\right]$ are determined in the first hand and then, in the second iteration, they are used to minimize Eq. (16). As aforesaid, convexity of the expected total discounted cost function has been proved for the single-item model. Convexity also exists when we are facing the two-item types scenario (i.e. $N=2$ ) where the obsolescence rates are equal for both item types, the discount rate is zero and the multipliers $k_{1}$ and $k_{2}$ are fixed to constant (see demonstration in Appendix D). However, the convexity structure of the general extended JRP total loss obsolescence model is not obvious.

\section{Numerical examples and results discussion}

An application developed in Excel VBA was used to run some test cases and obtain some results. All the test cases presented here involve three item types. The test cases were grouped in order to perform sensitivity analysis on several parameters of our JRP obsolescence model. Some of the test cases executed in the Excel VBA application were tested in MATLAB in order to compare and validate the obtained results. The results obtained in MATLAB were the same as the ones obtained with the Excel VBA application. The analysis is performed on a situation where a fictional company needs to systematically order and stock three item types subject to obsolescence from a single supplier. Several test cases are built in order to highlight the impact of the core features of our proposed model on the ordering policies, such as the obsolescence, time value of the money and JRP setup costs. Table 1 depicts the figures of each of the input parameters used to construct and run the several test cases. The values of the coordinated ordering are expressed in all the results depicted below by the optimal values of $E\left[V\left(S, T,\left(k_{1}, k_{2}, k_{3}\right)\right)\right]$ while the independent ordering values are the results obtained when each of the item types are ordered independently of each other. 
Table 1

Values of the parameters used to construct the test cases

\begin{tabular}{|c|c|c|c|}
\hline \multirow{2}{*}{ Parameter } & \multicolumn{3}{|c|}{ Values used in test cases } \\
\hline & Set 1 & Set 2 & Set 3 \\
\hline$\delta$ & 0.05 & 0.1 & 0.2 \\
\hline$\theta_{1}, \theta_{2} \theta_{3}$ & 0.2 & 0.3 & 0.5 \\
\hline $\mathrm{A}(\$)$ & 100 & 1000 & 10000 \\
\hline$a_{1}(\$)$ & 10 & 50 & 3000 \\
\hline$a_{2}(\$)$ & 15 & 70 & 800 \\
\hline$a_{3}(\$)$ & 10 & 70 & 500 \\
\hline$D_{1}$ (units) & 80 & 150 & 300 \\
\hline$D_{2}$ (units) & 200 & 400 & 600 \\
\hline$D_{3}$ (units) & 60 & 400 & 1200 \\
\hline$c_{1}(\$)$ & 2 & 4 & 6 \\
\hline$c_{2}(\$)$ & 3 & 8 & 12 \\
\hline$c_{3}(\$)$ & 1 & 10 & 30 \\
\hline (\$/unit/time) $h_{1}$ & 0.2 & 0.6 & 1.5 \\
\hline (\$/unit/time) $h_{2}$ & 0.3 & 1.2 & 3 \\
\hline (\$/unit/time) $h_{3}$ & 0.1 & 1.5 & 7.5 \\
\hline
\end{tabular}

\subsection{First group of test cases - analysis on obsolescence rates}

The first group includes six test cases with differences in the obsolescence rates and in the major setup cost. In the first three test cases the obsolescence rate is the same among the item types. The discount rate is 0.1 and the major set cost is 1000 . However, the obsolescence rates increase from test case one to test case three (from 0.2 to 0.5 ). Test cases four, five and six are the same as test cases one, two and three, but the major setup cost is zero. All the other parameters of the six test cases do not change and are set to parameter set 1 values depicted in Table 1 . The results of the six test cases are presented below in Table 2.

Table 2

Results of the first group of test cases

\begin{tabular}{ccccccc}
\hline Test Case & $\mathbf{1}$ & $\mathbf{2}$ & $\mathbf{3}$ & $\mathbf{4}$ & $\mathbf{5}$ & $\mathbf{6}$ \\
\hline$T^{*}$ & 2.73 & 2.38 & 1.83 & 0.50 & 0.43 & 0.35 \\
(multiplier) $k_{1}^{*}$ & 1 & 1 & 1 & 1 & 1 & 1 \\
(multiplier) $k_{2}^{*}$ & 1 & 1 & 1 & 1 & 1 & 1 \\
(multiplier) $k_{3}^{*}$ & 1 & 1 & 2 & 1 & 1 & 1 \\
$\left(\right.$ decimal units) $Q_{1}^{*}$ & 218.24 & 190.28 & 146.28 & 40.21 & 34.54 & 27.89 \\
\hline (decimal units) $Q_{2}^{*}$ & 545.60 & 475.70 & 365.69 & 100.53 & 86.36 & 69.73 \\
$\left(\right.$ decimal units) $Q_{3}^{*}$ & 163.68 & 142.71 & 219.41 & 30.16 & 25.91 & 20.92 \\
\hline Independent Ordering Cost $(\$)$ & 8815.50 & 7661.34 & 6421.39 & 3182.00 & 2443.22 & 1693.34 \\
\hline$(\$) E\left[V\left(S, T,\left(k_{1}, k_{2}, k_{3}\right)\right)\right]$ & 6520.12 & 5492.21 & 4341.07 & 3200.45 & 2458.72 & 1705.46 \\
\hline Savings $(\$)$ & 2295.38 & 2169.13 & 2080.31 & -18.45 & -15.50 & -12.12 \\
\hline
\end{tabular}

From test cases one, two and three, we can see that savings of the coordinated ordering decrease as long as the obsolescence rates increase. The base cycle between successive replenishments decreases and the order sizes decrease as well. In fact, these results make sense. The risk of obsolescence conducts to smaller lot sizes in order to prevent against high inventory levels when item types become suddenly obsolete. The smaller savings of the coordinated ordering associated with an increase of the risk of obsolescence can be justified by the correspondent smaller base cycle which implies more frequent orders to satisfy the demands and, thus, incurring more frequently into ordering costs. The progressively smaller order quantities associated with the increase in the obsolescence rates are in line with the results obtained by van Delft \& Vial (1996) with the singleitem type total obsolescence model.

The savings of test cases four, five and six are slightly negative due to the null value of the major setup cost. When the major setup cost is zero, the benefit from coordinated ordering vanishes mainly because of the expected discounted cost components with respect to $t>T$, i.e., $e^{-\delta T} E\left(\sum_{i=1}^{N-1} \Psi_{i}\right)$, of the total expected discounted costs expression of our proposed JRP obsolescence model. This is aligned with the common sense that it is better to order item types separately when we are not confronted with a major setup cost that is shared by ordering the different item types. 


\subsection{Second group of test cases - analysis on the major setup cost}

Here the test cases one, two and three are equal except for the major setup cost, which increases from test case one to test case three $(100,1000$, and 10000 , respectively). The obsolescence rates among the item types are the same (equal to 0.5 for all item types). The test cases four, five and six are equal to test cases one, two and three, respectively, but the obsolescence rates are different among the item types (equal to $0.2,0.3$ and 0.5 for item types one, two and three, respectively). All the other parameters consider the parameter set 1 presented in Table 1. The obtained results are depicted in Table 3.

Table 3

Results of the second group of test cases

\begin{tabular}{ccccccc}
\hline Test Case & $\mathbf{1}$ & $\mathbf{2}$ & $\mathbf{3}$ & $\mathbf{4}$ & $\mathbf{5}$ & $\mathbf{6}$ \\
\hline$T^{*}$ & 0.67 & 1.93 & 4.86 & 0.92 & 2.24 & 6.15 \\
$k_{1}^{*}$ (multiplier) & 1 & 1 & 1 & 1 & 2 & 2 \\
$k_{2}^{*}$ (multiplier) & 1 & 1 & 1 & 1 & 1 & 1 \\
$k_{3}^{*}$ (multiplier) & 2 & 2 & 2 & 1 & 1 & 1 \\
$Q_{1}^{*}$ (decimal units) & 53.46 & 154.05 & 389.16 & 73.86 & 358.67 & 984.27 \\
$Q_{2}^{*}$ (decimal units) & 133.65 & 385.13 & 972.91 & 184.65 & 448.34 & 1230.34 \\
$Q_{3}^{*}$ (decimal units) & 80.19 & 231.08 & 583.75 & 55.39 & 134.50 & 369.10 \\
\hline Independent Ordering Cost $(\$)$ & 2677.62 & 6666.35 & 36145.69 & 3905.05 & 8372.38 & 39034.50 \\
$E\left[V\left(S, T,\left(k_{1}, k_{2}, k_{3}\right)\right)\right](\$)$ & 2380.89 & 4581.22 & 16328.47 & 3571.05 & 6234.83 & 19189.66 \\
Savings $(\$)$ & 296.73 & 2085.13 & 19817.22 & 334.00 & 2137.54 & 19844.83 \\
\hline
\end{tabular}

The results allow us to conclude that the greater the major setup cost the greater the savings of the coordinated ordering. The base cycle between successive replenishments and the order sizes increase substantially with the increase of the major setup cost. Within our coordinated ordering context, this is aligned with an increase of the ratio $\frac{\sum \text { setup costs }}{\sum \text { holding costs }}$. In fact, when the setup costs (i.e. $A+\sum a_{i}$ ) are substantially higher than the holding costs (i.e. $\sum h_{i}$ ), the replenishments will probably occur less frequently (Silver et al., 2017). This is observed with test cases one, two and three, and with test cases four, five and six, where the major setup cost increases from test case to test case while the holding costs parameters do not vary.

Another interesting result relates the major setup cost and the expected lifetime of the item types. The greater values of the major setup cost in test cases three and six imply a base cycle that is greater than the expected lifetime of the considered item types, i.e., $\max (1 / 0.5,1 / 0.5,1 / 0.5)=2$ units of time in test case three and $\max (1 / 0.2,1 / 0.3,1 / 0.5)=5$ units of time in test case six.

\subsection{Third group of test cases - analysis on the minor setup costs}

The third group of test cases are equal to the test cases of group two, except for differences on the setup costs. The major setup cost is the same for all test cases (1000). The minor setup cost of item type 1 is set to 10, 50 and 3000 in the first three test cases, respectively. For item type 3, the minor setup cost is equal to $10,70,500$ in the test cases four, five and six, respectively. The minor setup costs of item type 2 do not vary across all test cases. Results obtained are presented in Table 4 .

Table 4

Results of the third group of test cases

\begin{tabular}{ccccccc}
\hline Test Case & $\mathbf{1}$ & $\mathbf{2}$ & $\mathbf{3}$ & $\mathbf{4}$ & $\mathbf{5}$ & $\mathbf{6}$ \\
\hline$T^{*}$ & 1.93 & 1.63 & 1.77 & 2.24 & 2.07 & 2.20 \\
$k_{1}^{*}$ (multiplier) & 1 & 2 & 3 & 2 & 2 & 2 \\
$k_{2}^{*}$ (multiplier) & 1 & 1 & 1 & 1 & 1 & 1 \\
$k_{3}^{*}$ (multiplier) & 2 & 2 & 2 & 1 & 2 & 2 \\
$Q_{1}^{*}$ (decimal units) & 154.05 & 260.25 & 424.36 & 358.67 & 331.81 & 351.95 \\
$Q_{2}^{*}$ (decimal units) & 385.13 & 325.31 & 353.64 & 448.34 & 414.76 & 439.94 \\
$Q_{3}^{*}$ (decimal units) & 231.08 & 195.19 & 212.18 & 134.50 & 248.86 & 263.96 \\
\hline Independent Ordering Cost $(\$)$ & 6666.35 & 6714.28 & 9977.13 & 8372.38 & 8437.72 & 8899.87 \\
$E\left[V\left(S, T,\left(k_{1}, k_{2}, k_{3}\right)\right)\right\rceil(\$)$ & 4581.22 & 4633.88 & 7981.28 & 6234.83 & 6307.04 & 6786.58 \\
Savings $(\$)$ & 2085.13 & 2080.40 & 1995.84 & 2137.54 & 2130.67 & 2113.29 \\
\hline
\end{tabular}

In a similar way as verified in the second group of test cases on the major setup cost, a progressive increase of the minor setup costs causes the same impact in the time between replenishments and the corresponding ordered quantities. However, the coordinated ordering savings walks in the opposite way, i.e., decreases with the increase of the minor setup cost. From test cases one, two and three we can observe the progressive increase of the item type 1 multiplier $k_{1}$ due to the progressive increase of the item type 1 minor setup cost in the test cases one, two and three $(1,2,3)$. The same can also be verified in the 
multiplier $k_{3}$ of item type 3 in test cases four, five and six $(1,2,2)$. The expected lower replenishment frequency of these two item types is explained by the increase of the minor setup cost, as it implies an increase of the ratio $\frac{\sum \operatorname{setup} \text { costs }}{\sum \text { holding costs }}$.

\subsection{Fourth group - analysis on the discount rates}

The test cases one, two and three are equal, except for the progressive increase of the discount. The obsolescence rates in these test cases have different values from item type to item type $(0.2,0.3$ and 0.5 , respectively). The major setup cost is always equal to 1000. All other parameters are equal and set to the parameter set 2 values depicted in Table 1 . The test cases four, five and six are equal to first three test cases, but the obsolescence rates are zero, i.e., the item types are not subject to obsolescence. Results are presented next (Table 5).

Table 5

Results of the fourth group of test cases

\begin{tabular}{ccccccc}
\hline Test Case & $\mathbf{1}$ & $\mathbf{2}$ & $\mathbf{3}$ & $\mathbf{4}$ & $\mathbf{5}$ & $\mathbf{6}$ \\
\hline$T^{*}$ & 0.74 & 0.74 & 0.67 & 2.42 & 1.70 & 1.19 \\
(multiplier) $k_{1}^{*}$ & 2 & 1 & 1 & 1 & 1 & 1 \\
(multiplier) $k_{2}^{*}$ & 1 & 1 & 1 & 1 & 1 & 1 \\
(multiplier) $k_{3}^{*}$ & 1 & 1 & 1 & 1 & 1 & 1 \\
$\left(\right.$ decimal units) $Q_{1}^{*}$ & 220.87 & 110.37 & 100.59 & 363.08 & 254.61 & 177.95 \\
\hline (decimal units) $Q_{2}^{*}$ & 294.50 & 294.32 & 268.24 & 968.21 & 678.95 & 474.54 \\
(decimal units) $Q_{3}^{*}$ & 294.50 & 294.32 & 268.24 & 968.21 & 678.95 & 474.54 \\
\hline Independent Ordering Cost $(\$)$ & 32322.29 & 29420.15 & 25244.27 & 187977.2 & 101263.7 & 56113.32 \\
\hline$(\$) E\left[V\left(S, T,\left(k_{1}, k_{2}, k_{3}\right)\right)\right]$ & 28942.67 & 26063.50 & 21877.53 & 176070.0 & 92429.59 & 49443.45 \\
Savings $(\$)$ & 3379.62 & 3356.65 & 3366.74 & 11907.17 & 8834.10 & 6669.87 \\
\hline
\end{tabular}

From results of the fourth group of test cases we can conclude that the greater the discount rate the lesser the savings of the coordinated ordering. The base cycle between successive replenishments and order quantities of the item types also decreases with the discount rate increase. This result is in line with the increase of the risk of obsolescence in the first group of test cases above mentioned. Hence, an increase in the discount rate implies smaller lot sizes and a decrease of the base cycle. The absence of obsolescence in test cases four, five and six implies significantly greater coordinated ordering savings when compared to test cases where item types are prone to obsolescence. This is aligned with the results of the first group of test cases.

\subsection{Fifth group - analysis on holding cost, minor setup cost, demand and unit cost parameters}

The fifth group includes seven test cases and takes the test case 1 as a basis for analysis with the other six test cases changing the following parameters: holding cost, demand and unit cost. In this way, test cases one, two and three are equal, except the progressive increase of the holding cost parameter; test cases one, four and five are equal, except the progressive increase of the demand parameter; and test cases one, six and seven are equal, with exception to the progressive increase of unit cost parameter. All the remaining parameters are set to the values of the parameter set 2 of Table 1 . Results are presented in Table 6.

Table 6

Results of the fifth group of test cases

\begin{tabular}{cccccccc}
\hline Test Case & $\mathbf{1}$ & $\mathbf{2}$ & $\mathbf{3}$ & $\mathbf{4}$ & $\mathbf{5}$ & $\mathbf{6}$ & $\mathbf{7}$ \\
\hline$T^{*}$ & 2.50 & 2.25 & 1.84 & 1.67 & 1.23 & 1.49 & 1.11 \\
$k_{1}^{*}$ (multiplier) & 1 & 1 & 1 & 1 & 1 & 1 & 1 \\
$k_{2}^{*}$ (multiplier) & 1 & 1 & 1 & 1 & 1 & 1 & 1 \\
$k_{3}^{*}$ (multiplier) & 1 & 1 & 1 & 1 & 1 & 1 & 1 \\
$Q_{1}^{*}$ (decimal units) & 200.04 & 180.28 & 146.83 & 251.15 & 367.65 & 119.27 & 88.57 \\
$Q_{2}^{*}$ (decimal units) & 500.09 & 450.70 & 367.07 & 669.73 & 735.30 & 298.19 & 221.42 \\
$Q_{3}^{*}$ (decimal units) & 150.03 & 135.21 & 110.12 & 669.73 & 1470.61 & 89.46 & 66.43 \\
\hline Independent Ordering Cost $(\$)$ & 7867.52 & 8317.83 & 9354.80 & 12511.24 & 18948.36 & 14686.77 & 22291.78 \\
$E\left[V\left(S, T,\left(k_{1}, k_{2}, k_{3}\right)\right)\right](\$)$ & 5729.73 & 6071.99 & 6845.32 & 9914.75 & 15801.21 & 11980.75 & 19089.51 \\
Savings $(\$)$ & 2137.79 & 2245.84 & 2509.48 & 2596.49 & 3147.15 & 2706.02 & 3202.26 \\
\hline
\end{tabular}


Taking the results of the fifth group into account, we can conclude the following:

- The greater the holding costs, the greater the savings of the coordinated ordering, but the lesser the time between successive replenishments and the ordering quantities of all the item types; the same behavior holds when we increase the unit cost parameters of the item types;

- The greater the demand, the greater the savings of the coordinated ordering and the quantities of all the item types, but the lesser the time between successive replenishments.

\section{Conclusion}

This article developed an extension of the JRP where the item types are prone to obsolescence. We started from a previous single item type total obsolescence model, which does not consider holding costs, that optimizes the total expected discounted cost criterion, and extended it in order to consider holding costs and to cope with multiple types of items under the JRP context. The demand and the obsolescence rates of the item types are constant. Obsolescence time of every item type is assumed to follow a negative exponential distribution, which means that the lifetimes of the different item types are uncertain.

The convexity of the single item obsolescence model with holding costs is proved, but the convexity of the JRP obsolescence model is not obvious. Nevertheless, we prove the convexity of the model in the particular case of two item types with equal obsolescence rates and where the integer multipliers variables of both item types are assumed a priori to be one.

Sensitivity analysis has been performed on model parameters in order to analyze their impact in the ordering policy and in the savings obtained with the coordinated ordering. As far as the impact of obsolescence is concerned, an increase of the risk of obsolescence, through the increase of the obsolescence rates, may turn out a decrease of the savings of the coordinated ordering, the time between successive replenishments as well as the ordering quantities. Regarding the time value of money, the same impact occurs when we face a progressive increase of the discount rate.

The expression of total expected discounted cost of the JRP obsolescence model has a number of cost terms which is dependent on the number of item types. This fact may have a significant impact on the number of needed calculations to determine the optimal value when the number of item types is large enough. We can decrease the number of calculations by removing components from the expression that converges to zero when the number of item types is a sufficiently large number.

\section{Acknowledgement}

This work has been funded by national funds through FCT - Fundação para a Ciência e a Tecnologia, I.P., Project UIDB/05037/2020.

\section{References}

Ai, X.-Y., Zhang, J.-L., \& Wang, L. (2017). Optimal joint replenishment policy for multiple non-instantaneous deteriorating items. International Journal of Production Research, 55(16), 4625-4642.

Andriolo, A., Battini, D., Grubbström, R. W., Persona, A., \& Sgarbossa, F. (2014). A century of evolution from Harris's basic lot size model: survey and research agenda. International Journal of Production Economics, 155, 16-38.

Arcelus, F. J., Pakkala, T. P. M., \& Srinivasan, G. (2002). A myopic policy for the gradual obsolescence problem with pricedependent demand. Computers \& Operations Research, 29(9), 1115-1127.

Arcelus, F. J., Pakkala, T. P. M., \& Srinivasan, G. (2006). The instant obsolescence problem with price-dependent demand. Information Systems and Operational Research, 44(4), 248-266.

Axsäter, S. (2015). Coordinated ordering. In C. C. Price, J. Zhu, \& F. S. Hillier (Eds.), Inventory Control (3rd ed., Vol. 225, pp. 121-145). Springer International Publishing Switzerland.

Bakker, M., Riezebos, J., \& Teunter, R. H. (2012). Review of inventory systems with deterioration since 2001. European Journal of Operational Research, 221(2), 275-284.

Bartels, B., Ermel, U., Sandborn, P., \& Pecht, M. G. (2012). Strategies to the prediction, mitigation and management of product obsolescence (A. P. Sage (ed.)). John Wiley \& Sons, Inc.

Bastos, L., Mendes, M., Nunes, D., Melo, A., \& Carneiro, M. (2017). A systematic literature review on the joint replenishment problem solutions: 2006-2015. Production, 27(e20162229), 1-11.

Berk, E., \& Gurler, U. (2017). Inventory theory. In J. Sengupta, Raghu; Gupta, Aparna; Dutta (Ed.), Decision Sciences Theory and Practice (pp. 349-411). CRC Press - Taylor \& Francis Group.

Cobbaert, K., \& Van Oudheusden, D. (1996). Inventory models for fast moving spare parts subject to "sudden death" obsolescence. International Journal of Production Economics, 44(3), 239-248.

Cohen, T., \& Yedidsion, L. (2015). The periodic joint replenishment problem is strongly NP-hard. Mathematics of Operations Research, 43(4).

Dagpunar, J. S. (1982). Formulation of a multi item single supplier inventory problem. Journal of the Operational Research Society, 33(3), 285-286.

Dohi, T., \& Osaki, S. (1995). Optimal inventory policies under product obsolescent circumstance. Computers \& Mathematics with Applications, 29(3), 23-30.

Emsermann, M., \& Simon, B. (2007). Optimal control of an inventory with simultaneous obsolescence. Interfaces, 37(5), 445-454. 
Goyal, S. K., \& Giri, B. C. (2001). Recent trends in modeling of deteriorating inventory. European Journal of Operational Research, 134(1), 1-16.

Harris, F. W. (1913). How many parts to make at once. Factory, The Magazine of Management, 10(2), 135-136, 152.

Holmbom, M., \& Segerstedt, A. (2014). Economic order quantities in production: from Harris to economic lot scheduling problems. International Journal of Production Economics, 155, 82-90.

Huang, C.-J. (2004). The convexity and bounds on optimal cycle time for the EOQ model with obsolescent items. Journal of Statistics and Management Systems, 7(2), 237-251.

Joglekar, P., \& Lee, P. (1993). An exact formulation of inventory costs and optimal lot size in face of sudden obsolescence. Operations Research Letters, 14(5), 283-290.

Khanlarzade, N., Yegane, B. Y., Kamalabadi, I. N., \& Farughi, H. (2014). Inventory control with deteriorating items: a stateof-the-art literature review. International Journal of Industrial Engineering Computations, 5(2), 179-198.

Khouja, M., \& Goyal, S. K. (2008). A review of the joint replenishment problem literature: 1989-2005. European Journal of Operational Research, 186(1), 1-16.

Khouja, M., Saydam, C., Vergara, F. E., \& Rajagopalan, H. (2006). Optimal inventory policy under continuous unit cost decrease and risk of sudden obsolescence. International Journal of Operations \& Quantitative Management, 12(2), 1-18.

Kouki, C., Babai, M. Z., Jemai, Z., \& Minner, S. (2016). A coordinated multi-item inventory system for perishables with random lifetime. International Journal of Production Economics, 181, 226-237.

Lee, F.-C., \& Yao, M.-J. (2003). A global optimum search algorithm for the joint replenishment problem under power-of-two policy. Computers \& Operations Research, 30(9), 1319-1333.

Li, C., Xu, Xi., \& Zhan, D. (2009). Solving joint replenishment problem with deteriorating items using genetic algorithm. Journal of Advanced Manufacturing Systems, 08(01), 47-56.

Masters, J. M. (1991). A Note on the effect of sudden obsolescence on the optimal lot size. Decision Sciences, 22(5), 11801186.

Menipaz, E. (1986). An inventory model with product obsolescence and its implications for high technology industry. IEEE Transactions on Reliability, 35(2), 185-187.

Nahmias, S. (1982). Perishable inventory theory: a review. Operations Research, 30(4), 680-708.

Nahmias, S. (2011). Perishable inventory systems. In International Series in Operations Research \& Management Science, Vol. 160 (1st ed.). Springer US.

Oguji, N. (2018). Strategies for managing excess and dead inventories: a case study of spare parts inventories in the elevator equipment industry. In Operations and Supply Chain Management: An International Journal (Vol. 11).

Pahl, J., \& Voß, S. (2014). Integrating deterioration and lifetime constraints in production and supply chain planning: a survey. European Journal of Operational Research, 238(3), 654-674.

Pierskalla, W. P. (1969). An inventory problem with obsolescence. Naval Research Logistics Quarterly, 16(2), $217-228$.

Porras, E., \& Dekker, R. (2008). A solution method for the joint replenishment problem with correction factor. International Journal of Production Economics, 113(2), 834-851.

Sandborn, P. (2013). Design for obsolescence risk management. Procedia CIRP, 11, 15-22.

Silver, E. A., Pyk, D. F., \& Thomas, D. J. (2017). Coordinated replenishments at a single stocking point. In Inventory and Production Management in Supply Chains (4th ed., Vol. 1, pp. 437-485). CRC Press - Taylor \& Francis Group.

Song, J.-S., \& Zipkin, P. H. (1996). Managing inventory with the prospect of obsolescence. Operations Research, 44(1), 215222.

Taft, E. W. (1918). The most economical production lot. The Iron Age, 101(18), 1410-1412.

Taleizadeh, A. A., Wee, H.-M., \& Jolai, F. (2013). Revisiting a fuzzy rough economic order quantity model for deteriorating items considering quantity discount and prepayment. Mathematical and Computer Modelling, 57(5-6), 1466-1479.

Thompson, H. E. (1975). Inventory management and capital budgeting: a pedagogical note. Decision Sciences, 6(2), 383398.

van Delft, C., \& Vial, J. P. (1996). Discounted costs, obsolescence and planned stockouts with the EOQ formula. International Journal of Production Economics, 44(3), 255-265.

Webb, I. R., Buzby, B. R., \& Campbell, G. M. (1997). Cyclical schedules for the joint replenishment problem with dynamic demands. Naval Research Logistics (NRL), 44(6), 577-589.

\section{Appendices}

Appendix A - Derivation of the holding costs expression

The holding costs, as expected at the beginning of the cycle, are given by equation (7):

$$
h \int_{0}^{T} e^{-\delta t}(D T-D t) \theta e^{-\theta t} d t=h \theta\left(\frac{D T}{\delta+\theta}+\frac{D\left(e^{-(\delta+\theta) T}-1\right)}{(\delta+\theta)^{2}}\right)
$$


Proof:

In the first step we determine the indefinite integral:

$$
\begin{aligned}
& h \int e^{-\delta t}(D T-D t) \theta e^{-\theta t} d t=h \theta D \int(T-t) e^{-(\delta+\theta) t} d t=h \theta D\left((T-t)\left(-\frac{e^{-(\delta+\theta) t}}{\delta+\theta}\right)-\int\left(-\frac{e^{-(\delta+\theta) t}}{\delta+\theta}\right)(-1) d t\right)= \\
& h \theta D\left(-\frac{(T-t) e^{-(\delta+\theta) t}}{\delta+\theta}-\int \frac{e^{-(\delta+\theta) t}}{\delta+\theta} d t\right)=h \theta D\left(-\frac{(T-t) e^{-(\delta+\theta) t}}{\delta+\theta}-\frac{\int e^{-(\delta+\theta) t} d t}{\delta+\theta}\right)=h \theta D\left(-\frac{(T-t) e^{-(\delta+\theta) t}}{\delta+\theta}-\frac{\int \frac{e^{u}}{-(\delta+\theta)} d u}{\delta+\theta}\right)= \\
& h \theta D\left(-\frac{(T-t) e^{-(\delta+\theta) t}}{\delta+\theta}+\frac{e^{u}}{(\delta+\theta)^{2}}\right)=h \theta D\left(-\frac{(T-t) e^{-(\delta+\theta) t}}{\delta+\theta}+\frac{e^{-(\delta+\theta) t}}{(\delta+\theta)^{2}}\right)
\end{aligned}
$$

In the second step we determine the definite integral:

$$
\begin{aligned}
h \int_{0}^{T} e^{-\delta t}(D T-D t) & \theta e^{-\theta t} d t=\left[h \theta D\left(-\frac{(T-t) e^{-(\delta+\theta) t}}{\delta+\theta}+\frac{e^{-(\delta+\theta) t}}{(\delta+\theta)^{2}}\right)\right]_{t=T}-\left[h \theta D\left(-\frac{(T-t) e^{-(\delta+\theta) t}}{\delta+\theta}+\frac{e^{-(\delta+\theta) t}}{(\delta+\theta)^{2}}\right)\right]_{t=0} \\
= & h \theta D\left(-0+\frac{e^{-(\delta+\theta) T}}{(\delta+\theta)^{2}}+\frac{T}{\delta+\theta}-\frac{1}{(\delta+\theta)^{2}}\right)=h \theta D\left(\frac{T}{\delta+\theta}+\frac{e^{-(\delta+\theta) T}}{(\delta+\theta)^{2}}-\frac{1}{(\delta+\theta)^{2}}\right) \\
= & h \theta\left(\frac{D T}{\delta+\theta}+\frac{D\left(e^{-(\delta+\theta) T}-1\right)}{(\delta+\theta)^{2}}\right),
\end{aligned}
$$

which was to be demonstrated.

Appendix B - Derivation of $\mathrm{E}[\mathrm{V}(\mathrm{T})]$ with holding costs

The expression of $E[V(T)]$ with holding costs is given by equation (9):

$$
E[V(T)]=\frac{A+a+c D T+h \theta\left(\frac{D T}{\delta+\theta}+\frac{D\left(e^{-(\delta+\theta) T}-1\right)}{(\delta+\theta)^{2}}\right)}{\left(1-e^{-(\delta+\theta) T}\right)}
$$

Proof:

Let $L$ be the negative exponential random variable of the item lifetime such that $L \sim \operatorname{Exp}(\theta)$ and, to simplify the expressions, let $H=h \theta\left(\frac{D T}{\delta+\theta}+\frac{D\left(e^{-(\delta+\theta) T}-1\right)}{(\delta+\theta)^{2}}\right)$.

In a first moment, consider $V(T)$ without the impact of obsolescence, but with the expected holding costs $H$ :

$$
V(T)=\sum_{k=0}^{\infty}(A+a+c D T+H) e^{-\delta k T}
$$

Now, taking expectation and considering the impact the obsolescence in Eq. (B.2), multiplying each cost component by the probability of survival of the item type, the expected discounted cost along the infinite planning horizon is given by:

$$
\begin{gathered}
E[V(T)]=\sum_{m=0}^{\infty}((A+a+c D T+H) P[L>m T]) e^{-\delta m T}=\sum_{m=0}^{\infty}(A+a+c D T+H) e^{-\delta m T} e^{-\theta m T}= \\
=(A+a+c D T+H)+e^{-(\theta+\delta) T}\left(\sum_{m=0}^{\infty}(A+a+c D T+H) e^{-(\theta+\delta) m T}\right) \\
=(A+a+c D T+H)+e^{-(\theta+\delta) T}(E(V(T))) \Leftrightarrow \\
E[V(T)]=\frac{A+a+c D T+H}{1-e^{-(\theta+\delta) T}}
\end{gathered}
$$

which was to be demonstrated.

\section{Appendix C - Convexity of E[V(T)]}

$E[V(T)]$ is convex and, thus, has a global minimum in $\mathbb{R}^{+}$.

Proof: 
The expression of $E[V(T)]$ excluding the holding costs, i.e. $\frac{A+a+c D T}{\left(1-e^{-(\delta+\theta) T}\right)}$ ), is convex (see Huang, 2004). In this way, to demonstrate the convexity of $E[V(T)]$, it is enough to prove the convexity of the expression $\frac{h \theta\left(\frac{D T}{\delta+\theta}+\frac{D\left(e^{-(\delta+\theta) T}-1\right)}{(\delta+\theta)^{2}}\right)}{\left(1-e^{-(\theta+\delta) T}\right)}$. This expression can be rewritten as $\frac{\frac{h \theta D T}{\delta+\theta}}{\left(1-e^{-(\theta+\delta) T}\right)}+\frac{\frac{h \theta D\left(e^{-(\delta+\theta) T}-1\right)}{(\delta+\theta)^{2}}}{\left(1-e^{-(\theta+\delta) T}\right)}$. The first term is convex as it is simply a particular case of the $E[V(T)]$ without the holding costs where $A+a=0$ and $c=\frac{h \theta}{\delta+\theta}$.

Ignoring now, without loss of generality, the constant terms of the numerator of the second term, we only need to prove that $J(T)=\frac{e^{-(\delta+\theta) T}}{\left(1-e^{-(\delta+\theta) T}\right)}$ is convex.

The derivative of $J(T)$ is $J^{\prime}(T)=\left(\frac{e^{-(\delta+\theta) T}}{\left(1-e^{-(\delta+\theta) T}\right)}\right)^{\prime}=\left(\frac{1}{\left(e^{(\delta+\theta) T}-1\right)}\right)^{\prime}=-\frac{(\delta+\theta) e^{(\delta+\theta) T}}{\left(e^{(\delta+\theta) T}-1\right)^{2}}$ and the second derivative is $J^{\prime \prime}(T)=$ $\left(-\frac{(\delta+\theta) e^{(\delta+\theta) T}}{\left(e^{(\delta+\theta) T}-1\right)^{2}}\right)^{\prime}=\frac{(\delta+\theta)^{2} e^{(\delta+\theta) T}\left(e^{(\delta+\theta) T}+1\right)}{\left(e^{(\delta+\theta) T}-1\right)^{3}}$. Because $J^{\prime \prime}(T)>0$ for $T>0$, then $J(T)$ is convex, which was to be demonstrated.

Appendix D - Convexity of JRP obsolescence model with restricted conditions - a case with two item types

Let us consider the case where the number of item types is two $(N=2$ and $S=(1,2))$. If $\theta_{1}=\theta_{2}=\theta, \delta=0$, and $k_{1}$ and $k_{2}$ are fixed constants a priori, then the total expected discounted cost function $E[V((1,2), T,(1,1))]$ is convex. Therefore, assuming the integer multipliers as fixed constants implies that the total expected discounted cost expression is converted into a one-variable function, i.e., a function where the only decision variable is the base cycle $T$.

Proof:

Let $S=(1,2), \theta_{1}=\theta_{2}=\theta, \delta=0$ and, for the sake of simpler formulas, $k_{1}=k_{2}=1$. Then, $E[V((1,2), T,(1,1))]$ can be written as:

$$
\begin{aligned}
& E[V((1,2), T,(1,1))] \\
& =\frac{A+\sum_{i=1}^{2}\left(a_{i}+c_{i} d_{i} T+h_{i} \theta\left(\frac{D_{i} T}{\theta}+\frac{D_{i}\left(e^{-\theta T}-1\right)}{\theta^{2}}\right)\right)}{\left(1-e^{-2 \theta T}\right)}+\frac{\sum_{\substack{i=1 \\
i \neq j}}^{2} \sum_{\substack{j=1 \\
j \neq i}}^{2} E^{*}[V((i), \Gamma((i)), 1)]\left[e^{-\theta T}\left(1-e^{-\theta T}\right)\right]}{\left(1-e^{-2 \theta T}\right)}
\end{aligned}
$$

Convexity of the single item type model has been already demonstrated in A.3. Now, comparing the expression $E[V((1,2), T,(1,1))]$ with the expression $E(V(T))$ of the single item type model, where convexity holds, we just need to demonstrate that the component $H(T)=\frac{E^{*}[V((i), \Gamma((i)), 1)]\left[e^{-\theta T}\left(1-e^{-\theta T}\right)\right]}{\left(1-e^{-2 \theta T}\right)}$ is convex in order to ensure $E[V((1,2), T,(1,1))]$ convexity. The other terms are convex by means of appendix $C$. Therefore, using MATLAB, we can verify that the second derivative of $H(T)$ is strictly positive, i.e. $H(T)^{\prime \prime}=\frac{E^{*}[v((i), \Gamma((i)), 1)] * \theta^{2} * e^{\theta T}\left(e^{\theta T}-1\right)}{\left(e^{\theta T}+1\right)^{3}}>0, \forall_{T>0}$, ensuring which was to be demonstrated.

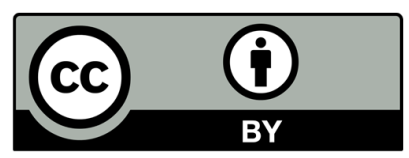

(C) 2022 by the authors; licensee Growing Science, Canada. This is an open access article distributed under the terms and conditions of the Creative Commons Attribution (CCBY) license (http://creativecommons.org/licenses/by/4.0/). 